\title{
A Satisfação de Estudantes Universitários com o Curso de Ensino Superior
}

\author{
Adriana Benevides Soares ${ }^{1,2}$ \\ ${ }^{1}$ Universidade Salgado de Oliveira, Niterói, RJ, Brasil. \\ ${ }^{2}$ Universidade do Estado do Rio de Janeiro, RJ, Brasil. \\ Isabela da Silva Rodrigues ${ }^{1}$ \\ ${ }^{1}$ Universidade Salgado de Oliveira, Niterói, RJ, Brasil.
}

\author{
Giselle Glória Balbino dos Santos ${ }^{1}$ \\ ${ }^{1}$ Universidade Salgado de Oliveira, Niterói, RJ, Brasil. \\ Claudio de Almeida Lima ${ }^{1}$ \\ ${ }^{1}$ Universidade Salgado de Oliveira, Niterói, RJ, Brasil.
}

\begin{abstract}
Resumo: Este estudo exploratório qualitativo objetivou identificar as características que compõem a satisfação dos estudantes universitários com o curso superior. A amostra foi composta por 78 alunos de diferentes cursos de instituições públicas e privadas situadas no estado do Rio de Janeiro. Na coleta de dados, foi utilizado um questionário específico construído especialmente para este estudo, baseado na literatura pertinente. Para explorar os dados, foi realizada a análise do corpus textual por meio do software Iramuteq (versão 0.7 alpha 2). Os resultados evidenciaram características de satisfação e insatisfação dos alunos. A satisfação foi especialmente proporcionada pela figura do docente, indicando o papel dele como facilitador no desenvolvimento do conhecimento e das habilidades profissionais dos estudantes. A insatisfação se relacionou principalmente ao não oferecimento de diversos elementos pelo curso, dentre os quais destacam-se o papel do docente, a oportunidade de vivenciar aspectos práticos da profissão, a estrutura da Instituição e a qualidade da informação fornecida. Os achados oferecem subsídios para que as universidades possam melhorar suas práticas, elevando a qualidade dos cursos.
\end{abstract}

Palavras-chave: Satisfação, Ensino superior, Estudantes universitários.

\section{The Satisfaction of University Students with Higher Education Courses}

\begin{abstract}
This qualitative exploratory study sought to identify the characteristics that make up the satisfaction and dissatisfaction of university students. The sample consisted of 78 students from different higher education courses, from public and private institutions located in the state of Rio de Janeiro. Data was collected using a questionnaire constructed specifically for this study, based on the relevant literature, and explored by textual corpus analysis performed on Iramuteq software (version 0.7 alpha 2). Results show that satisfaction was represented mainly by the professor, indicating their role as facilitator in the development of knowledge and professional skills. Dissatisfaction was mainly related to the course not offering several elements, such as the role of the professor, the opportunity to experience practical aspects of the profession, the institution's structure, and the quality of the information provided. Our findings offer support for universities to improve their practices, raising the quality of courses.
\end{abstract}

Keywords: Satisfaction, Higher Education, University students.

\section{La Satisfacción de los Universitarios con el Curso de Educación Superior}

Resumen: Este estudio exploratorio y cualitativo pretende identificar las características que conforman la satisfacción de los estudiantes universitarios con el curso superior. La muestra 
estuvo compuesta de 78 alumnos, de diferentes cursos en instituciones públicas y privadas ubicadas en el estado de Río de Janeiro. Para la recopilación de datos, se utilizó un cuestionario específico construido especialmente para este estudio a partir de la literatura pertinente. Para explorar los datos se realizó el análisis del corpus textual por medio del software Iramuteq (versión 0.7 alfa 2). Los resultados evidenciaron características de satisfacción e insatisfacción de los alumnos. La satisfacción fue especialmente representada por la figura del docente, indicando el papel de este como facilitador en el desarrollo del conocimiento y habilidades profesionales. La insatisfacción se relacionó principalmente con la falta de diversos elementos ofrecidos por el curso, entre los principales estaban el papel del docente, el ofrecimiento de oportunidad de vivir aspectos prácticos relacionados con la profesión, la estructura de la Institución y la calidad de la información suministrada. Los hallazgos proporcionan subsidios para que las universidades puedan mejorar sus prácticas, elevando la calidad de los cursos.

Palabras clave: Satisfacción, Educación superior, Estudiantes universitarios.

\section{Introdução}

O estudante, ao ingressar na universidade, vivencia algum desconforto frente a diferentes cobranças e a novas exigências demandadas pelo contexto acadêmico. As pesquisas que investigam a satisfação dos universitários fornecem informações sobre a diversidade de influências que acarretam problemas ou sucesso no processo educacional, munindo as universidades com subsídios para melhorarem a qualidade do ensino, por meio do aprimoramento dos professores, da infraestrutura, dos currículos e de programas educacionais (Hirsch, 2015).

Para Hirsch, Barlem, Barlem, Silveira e Mendes (2015), a satisfação com o curso compreende a percepção do sujeito diante do alcance de suas expectativas acadêmicas, ou seja, a sensação de sucesso conquistado em relação ao desempenho educacional. Compreende-se essa satisfação dos alunos como um elo entre o estudante e a Instituição de Ensino Superior (IES), tendo em vista o grau de satisfação que o estudante demonstra em relação à qualidade do ensino e aos serviços fornecidos por ela (Alcântara, Luiz, Ferreira, \& Teodoro, 2012). Dessa forma, a variável satisfação com o curso predomina nos estudos que têm como público-alvo os estudantes do Ensino Superior, uma vez que possibilita identificar seu julgamento sobre a realidade percebida na universidade (Souza, \& Reinert, 2010).

Diferentes fatores podem influenciar a satisfação dos discentes com o curso: o método utilizado pelos professores, a qualidade do ensino, a estrutura física da instituição, como em salas de aula e laboratórios (Camargos, Camargos, \& Machado, 2006; Douglas, Douglas, \& Barnes, 2006; Fernandes, Silva, Ravena,
Martins, \& Gomes, 2017). Segundo Gomes, Dagostini e Cunha (2013), a satisfação com o curso é um dos aspectos fundamentais para a manutenção dos estudantes e para uma imagem positiva da universidade.

Em diversos estudos, a investigação da satisfação de universitários com o curso apresenta-se como um elemento fundamental na avaliação da eficácia institucional e dos contextos educativos, proporcionando às IES a compreensão da interação entre o estudante e a universidade, assim como a reestruturação de sua organização visando se adequarem às necessidades dos alunos (Ahmed, Sarwar, \& Riaz, 2018; Bordin et al., 2016; Campos, Martins, \& Neto, 2014; Carmo, Silva, Miranda, Rabelo, \& Guimarães, 2011; Fadel et al., 2018; Ramos et al., 2015). De forma geral, percebe-se que avaliar a satisfação com o curso possibilita insumos para a melhoria dos programas e serviços educacionais, elevando, assim, a eficácia do processo educativo. Dessa forma, a literatura fornece instrumentos que foram desenvolvidos para medir a satisfação geral dos estudantes, por meio de suas vivências durante a formação acadêmica (Pinto, Quadros, Cruz, \& Conrad, 2016), ou seja, as medidas disponíveis avaliam diferentes aspectos relacionados ao construto e não à satisfação com o curso especificamente.

A Escala de Satisfação Acadêmica (ESA), desenvolvida por Martins (1998), considera cinco dimensões e três fatores (Aplicabilidade, Formação e Possibilidade de desenvolvimento). Um segundo instrumento é o Questionário de Satisfação Acadêmica (QSA) (Soares, Vasconcelos, \& Almeida, 2002) que objetiva avaliar o grau de satisfação dos estudantes associado a diversos aspectos de sua experiência universitária. Propõe-se a avaliar três dimensões: Social, Institucional e 
Curricular. Um terceiro instrumento é a Escala de Satisfação com a Experiência Acadêmica, desenvolvida por Schleich, Polydoro e Santos (2006), que contempla aspectos relacionados ao curso, à instituição e às oportunidades de desenvolvimento pessoal e profissional, assim como características relacionadas ao contexto acadêmico. Considerando as características desses instrumentos, pode-se observar que avaliam a satisfação acadêmica e medem a satisfação com o curso apenas como uma dimensão da escala.

Estudos empíricos também foram desenvolvidos e avaliaram a satisfação dos graduandos em relação ao curso superior, apontando estratégias que podem melhorar a aprendizagem dos estudantes, como o suporte a professores e coordenadores de cursos (Cunha, Gomes, \& Beck, 2016; Gomes et al., 2013; Hirsch et al., 2016; Souza, \& Reinert, 2010).

O estudo desenvolvido por Cunha et al. (2016) com 257 alunos de diferentes períodos do curso de Ciências Contábeis de universidades públicas de Santa Catarina, buscou avaliar a satisfação geral dos estudantes. Nos resultados, expõe uma correlação forte e positiva entre a Organização do Curso e o Interesse do Estudante, o que indica que a dimensão Organização do Curso relaciona-se com o conteúdo das demais disciplinas e que a Organização e a Estrutura do Curso junto ao coordenador possibilitam o bom desenvolvimento do curso, fomentando o Interesse do Estudante por meio da atenção do aluno em sala de aula e participação nas discussões. As Demandas do Curso e o Interesse dos Estudantes apontaram que as demandas são adequadas em relação à quantidade de conteúdos ministrados na disciplina, ao tempo utilizado para o desenvolvimento do programa, ao encadeamento curricular e à adequação das atividades acadêmicas.

Gomes et al. (2013) realizaram um estudo que buscou avaliar os determinantes da satisfação geral dos alunos. Participaram 212 graduandos do curso de Ciências Contábeis de uma faculdade do Sudoeste do Paraná. A partir da mesma teoria utilizada no estudo de Cunha et al. (2016), o modelo proposto apresenta três fatores: o primeiro inclui variáveis sobre a Dimensão do Curso, Organização do Curso e Satisfação Geral. O segundo é constituído por variáveis relativas à dimensão Envolvimento do Professor e o terceiro tem relação com a dimensão Envolvimento do Estudante, que diz respeito ao interesse do discente em aprender. Os resultados demonstraram que a satisfação geral com o curso apresentou uma média de 4,45 , que é considerada baixa, uma vez que a escala varia até sete. Observou-se que a maneira como o docente ministra o conteúdo didático em sala de aula é um dos determinantes da satisfação do estudante.

A análise da avaliação de um curso de graduação foi objeto da investigação realizada por Souza e Reinert (2010), que buscaram conhecer os fatores que originam a satisfação e a insatisfação na percepção dos estudantes, além de comparar os resultados entre as modalidades de ensino presencial e a distância. Por meio da Análise do Conteúdo, os resultados apontaram que, na avaliação dos estudantes, os fatores essenciais que definem a satisfação e a insatisfação com o curso estão relacionados com a estrutura curricular, o corpo docente e o ambiente social. Observou-se diferenças significativas na comparação entre as modalidades de ensino pesquisadas. Segundo a avaliação dos cursos presenciais, os fatores essenciais de satisfação foram a estrutura curricular e o corpo docente. No curso à distância, os mesmos fatores foram encontrados, porém, o corpo docente figurou em primeiro lugar, seguido da estrutura curricular. Verificou-se diferenças expressivas nas duas modalidades de ensino quanto à satisfação com o corpo docente. No curso presencial, a indicação de satisfação se referiu a um professor especificamente, enquanto no curso a distância a didática dos professores foi o aspecto mais satisfatório. Quanto à insatisfação com o curso, na modalidade presencial, o corpo docente e a estrutura curricular se destacaram, enquanto na modalidade à distância, foram considerados insatisfatórios o corpo docente e as características do curso.

Hirsch et al. (2015) buscaram identificar, em um estudo transversal com 123 estudantes deEnfermagem de uma universidade pública do sul do país, os preditores e fatores que se relacionam à satisfação dos estudantes com as atividades de currículo e ensino, interação social/profissional e ambiente de aprendizagem do curso de graduação. Os resultados evidenciaram que a dimensão currículo e ensino são as mais relevantes para a satisfação na percepção dos estudantes, seguida da dimensão ambiente de aprendizagem e interações sociais/profissionais. Ressalta-se que, na percepção dos graduandos, as questões referentes a capacitação do docente, utilização de metodologias apropriadas, didática atualizada, disposição dos conteúdos disciplinares e coerência das disciplinas 
foram as que propiciaram maior satisfação ou insatisfação acadêmica. Compreende-se, com esse estudo, a necessidade de se aperfeiçoar os aspectos educativos e formativos, especialmente com investimentos no currículo e no ensino visando contribuir para uma experiência acadêmica satisfatória.

Pode-se também apontar a experiência do estágio como uma vivência significativa de avaliação do curso e uma possibilidade de desenvolvimento de carreira. Santos, Goulart, Miyoshi e Santos (2014) buscaram analisar a confiabilidade e a validade interna de um questionário de satisfação com o estágio aplicado prospectivamente a alunos do sexto ano de Medicina que tinham frequentado o estágio de Neonatologia em uma universidade pública. Os resultados obtidos indicam a importância dos cenários de práticas que, associados ao programa teórico, auxiliem na melhoria do processo ensino-aprendizagem e, por consequência, levem a uma maior satisfação com o curso. A unidade curricular foi bem avaliada, de forma que a satisfação com o estágio foi positivamente correlacionada ao desempenho do estudante.

Alguns desses estudos também relatam características da insatisfação acadêmica, entendendo que é gerada pelo desencontro entre as expectativas dos alunos e o que a IES podem oferecer, o que tem como resultados baixo desempenho do graduando, dificuldade de adaptação à vida acadêmica, decepção com a profissão e até o abandono do curso (Souza, \& Reinert, 2010). Hirsch et al. (2015) apontam que a situação de insatisfação diária pode ocasionar desequilíbrio emocional como nervosismo, irritabilidade, impaciência e comportamento de retração, acarretando apatia, desmotivação quanto ao curso, estresse e até a desistência da futura profissão.

Desse modo, o presente estudo busca identificar as características que compõem a satisfação dos estudantes universitários com o curso superior, entendendo que as IES são entidades que possuem condições de transformar vidas fomentando o desenvolvimento do indivíduo (Silva, 2015).

\section{Método}

\section{Participantes}

Participaram deste estudo 78 estudantes universitários com idades de 19 a 50 anos ( $\mathrm{M}=25,09$ e DP=5,65), dos quais 27 (34,6\%) eram homens e $51(65,4 \%)$ eram mulheres. Foi utilizado o critério de saturação teórica para delimitar o tamanho da amostra (Nascimento et al., 2018). Em relação aos períodos, dois participantes $(2,6 \%)$ eram do terceiro período, $15(19,2 \%)$ do quarto, $13(16,7 \%)$ do quinto, $13(16,7 \%)$ do sexto, 16 (20,5\%) do sétimo, nove $(11,5 \%)$ do oitavo, três $(3,8 \%)$ do nono e sete $(9 \%)$ do $10^{\circ}$. Dentre os estudantes, $37(47,4 \%)$ eram de IES privadas e 41 (52,6\%) de IES públicas. Em relação ao estado civil, 66 (84,6\%) eram solteiros, 11 (14,1\%) casados e um (1,3\%) não respondeu. Do total da amostra, 21 (26,9\%) eram da área Social Aplicada, 20 (25,6\%) da Saúde, 19 (24,4\%) de Humanas e 18 (23,1\%) de Exatas. Em relação à classe social, quatro $(5,1 \%)$ eram da classe social A, oito $(10,3 \%)$ da B1, $25(32,1 \%)$ da B2, $29(37,2 \%)$ da $\mathrm{C} 1$, nove $(11,5 \%)$ da C2 e três $(3,8 \%)$ da D/E, conforme indicadores da Associação Brasileira de Empresas de Pesquisa (ABEP, 2018) que identificam as classes sociais a partir de itens de bens de consumo e do nível de escolaridade do chefe da família.

\section{Instrumentos}

Foi aplicado um questionário elaborado especificamente para o estudo e construído com base na literatura sobre a percepção dos estudantes em relação à satisfação com o curso (Schleich, Polydoro, \& Santos, 2006; Soares, Vasconcelos, \& Almeida, 2002). O questionário é constituído por 18 perguntas, das quais, neste estudo, foram analisadas apenas duas questões, as de caráter mais geral, referentes ao que os estudantes consideram mais satisfatório e insatisfatório, respectivamente. Utilizou-se também um Questionário Sociodemográfico para caracterizar a amostra coletando informações sobre: sexo, idade, estado civil, área de formação, período e classe social segundo critério de classificação econômica (ABEP, 2018).

\section{Procedimentos éticos}

Este estudo foi aprovado no Comitê de Ética em pesquisa conforme o CAAE 66060117.6.0000.5289 em 28 de março de 2017. Os objetivos da pesquisa foram explicados a todos os participantes que assinaram o Termo de Consentimento Livre e Esclarecido, garantindo-se o sigilo de sua identidade e o direito de retirar sua permissão de participação a qualquer momento.

\section{Procedimentos de coleta de dados}

Aplicou-se o questionário predominantemente de forma coletiva, embora alguns tenham tido aplicação individual. Foram coletados nos momentos em 
que os alunos estavam esperando o início das aulas. A coleta ocorreu em vários momentos, em diferentes IES públicas e privadas do estado do Rio de Janeiro. Os estudantes responderam a um formulário com questões sociodemográficas e, em seguida, ao questionário de satisfação com o curso.

\section{Procedimentos de análise de dados}

Foi realizada a análise das palavras do corpus textual por meio do software Iramuteq (versão 0.7 alpha 2). As respostas dos participantes foram organizadas em dois temas: a insatisfação com o curso e a satisfação com o curso. Neste estudo, optou-se por utilizar a análise lexical, que tem por unidade as palavras que são identificadas e quantificadas em termos de frequência e posição no texto (Justo, \& Camargo, 2014). A análise de similitude é uma ferramenta que consiste na apresentação de um grafo que representa a ligação entre as palavras do corpus textual. É baseada na teoria dos grafos e proporciona a identificação das coocorrências entre as palavras e indica sua conexão (Camargo, \& Justo, 2013). A partir disso, é possível compreender temas de relativa importância, sua estrutura de construção e como eles se relacionam, indicando as conexões entre as palavras.

\section{Resultados}

Na Figura 1 são apresentados os resultados da análise do corpus denominado Satisfação, do qual emergiram 753 ocorrências (palavras, formas ou vocábulos), das quais 308 são palavras distintas e 216 têm uma única ocorrência. Nessa imagem, são apresentados os resultados alusivos à temática Satisfação com o curso. Observa-se que a palavra central "professor" (frequência 25) tem maior importância no conteúdo do texto. Isso pode ser exemplificado em algumas respostas em relação ao que os alunos consideram mais satisfatório em seu curso: "O alto nível dos meus professores” (Participante 8, sexo feminino, Educação Física, julho de 2018); "A troca de experiências com amigos de professores do curso e algumas práticas que me estimulam junto ao meu ideal" (Participante 50, sexo feminino, Pedagogia, julho de 2018); "A oportunidade de aprender com professores muito capacitados" (Participante 55, sexo feminino, Psicologia, julho de 2018); e "A qualidade de ensino da maioria dos professores" (Participante 4, sexo masculino, Administração, julho de 2018).

Da palavra professor partem "estágio" (frequência=12) - que pode ser observada na resposta: "Considero mais satisfatório as diversas possibilidades de adquirir conhecimentos da minha área de atuação, através de eventos, palestras, projetos de extensão, estágio" (Participante 17, sexo feminino, Educação Física, julho de 2018) - e "curso" (f=13), palavras que possuem importâncias semelhantes, mas se contrapõem quanto ao sentido. Outras palavras em destaque são "aprender" (frequência=8), "prático" (frequência=7) e "conhecimento" (frequência=7), que foram observadas na fala: "Adquirir conhecimento para cuidar da saúde das pessoas..." (Participante 22, sexo feminino, Odontologia, julho de 2018), e "disciplina” (frequência=7), observada na fala: "O conteúdo das disciplinas com professores preocupados com o sucesso dos alunos" (Participante 32, sexo masculino, Direito, julho de 2018).

Da análise do corpus denominado Insatisfação, emergiram 1121 ocorrências (palavras, formas ou vocábulos), das quais 443 são palavras distintas e 300 têm uma única ocorrência. Na Figura 2, apresenta-se os resultados gerados pelo Iramuteq alusivos à temática Insatisfação com o curso. Observa-se três ramificações principais que partem da palavra "falta" (frequência=28) para outras que apresentam expressão significativa. No ramo à direita, visualiza-se as palavras "professor" (frequência=22) e "aluno" (frequência=19), em complementaridade ao ramo da esquerda, em que é possível observar as palavras "prático" (frequência=17) e "matéria" (frequência=15). Abaixo da palavra "falta", ainda é possível identificar outras palavras em destaque, como "curso" (frequência=11), "aula" (frequência=10) e "não" (frequência=18). Falas extraídas dos questionários referenciam essas análises: "A grade curricular não atende às demandas do mercado totalmente, pois leva o aluno muito tarde ao campo prático, prejudicando sua experiência" (Participante 70, julho de 2018); "Não há um espaço de convivência e lazer satisfatórios" (Participante 3, julho de 2018). 
Psicologia: Ciência e Profissão 2021 v. 41, e220715, 1-12.

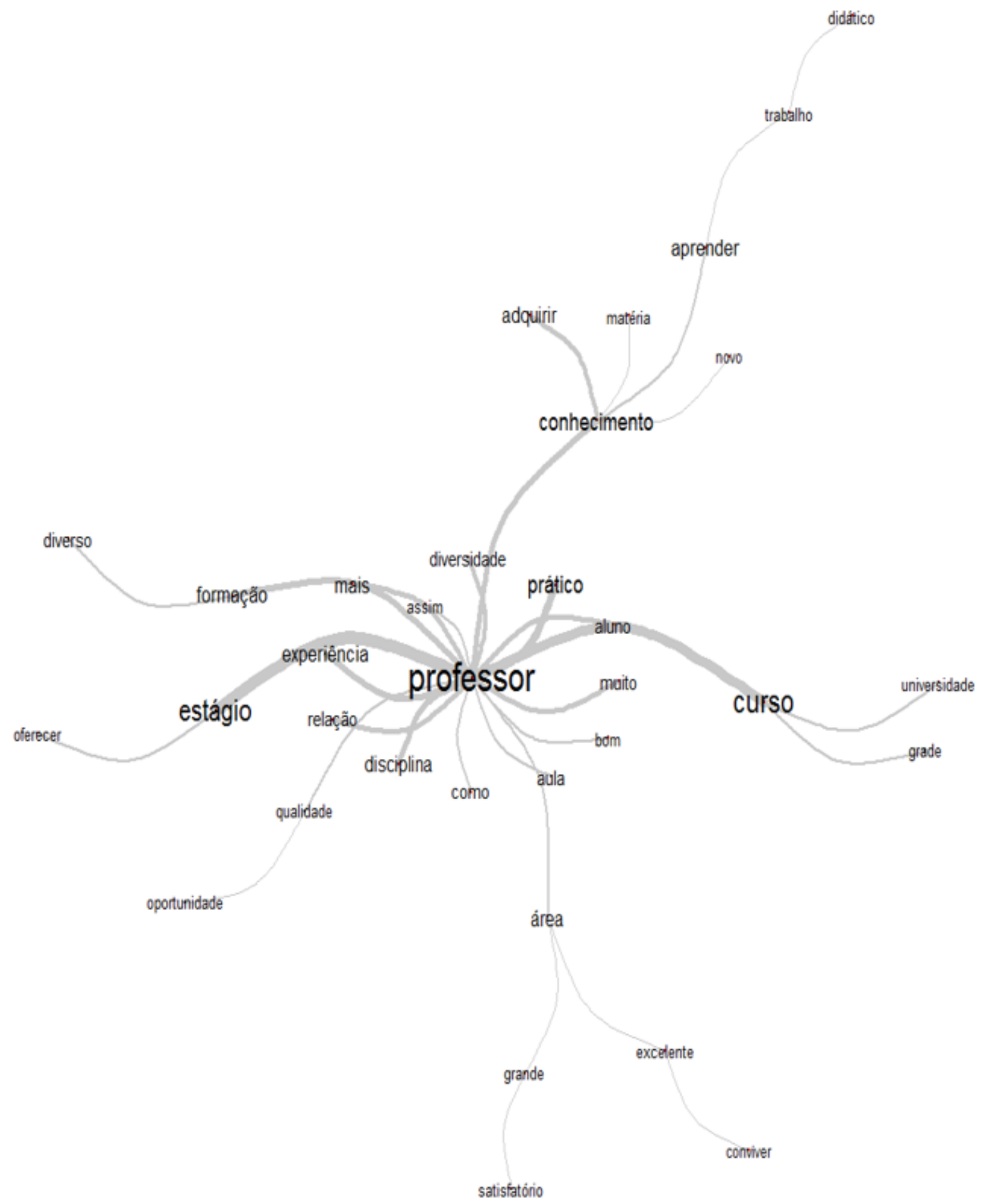

Figura 1

Grafo Satisfação com o curso. 


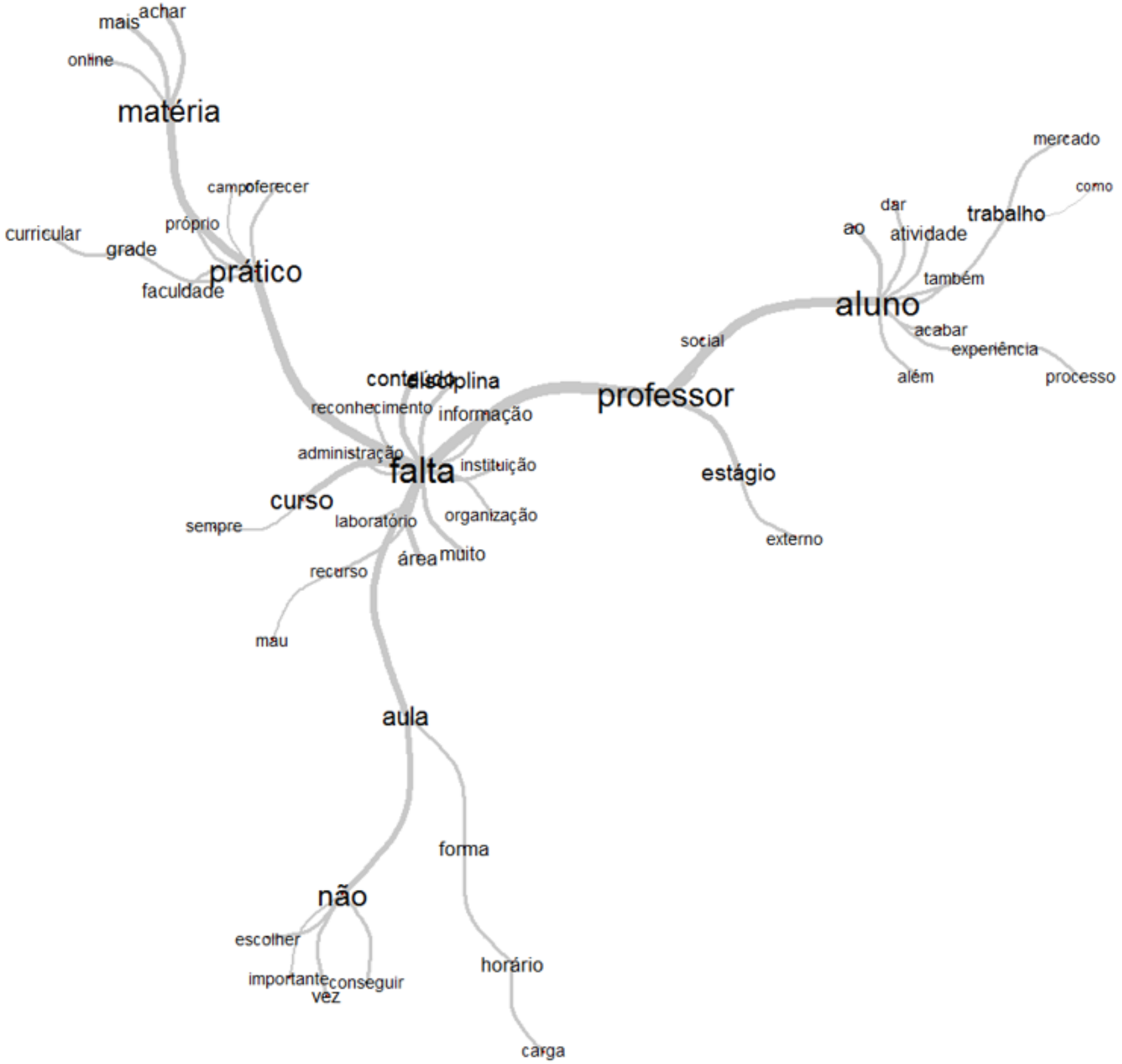

Figura 2

Grafo Insatisfação com o curso.

\section{Discussão}

Como elementos de satisfação dos alunos, o papel do professor assumiu destaque principal na avaliação do estudante. É possível identificar a importância do professor não só como facilitador do conhecimento, como também aquele que auxilia o aluno a fazer a transição do conteúdo teórico para o prático. Dessa forma, o professor é aquele que apoia o aluno no desenvolvimento das habilidades profissionais por meio dos estágios e de suas próprias experiências, que são importantes para a futura atuação no mercado de trabalho. De forma semelhante, Ahmed et al. (2018), em seu estudo com estudantes universitários, apontaram aspectos como a experiência do professor, a atenção dada aos alunos, as competência e atualização do conhecimento e o ambiente de ensino como aspectos associados à satisfação discente. Bordin et al. (2016) observaram que maiores índices de satisfação estavam relacionados a fatores associados às relações sociais, sobretudo entre discentes e os professores. Gomes et al. (2013) também ressaltaram que a maneira como o docente desenvolve o conteúdo didático, além de seu 
envolvimento nas aulas, tem relevância na satisfação dos alunos. Outros estudos (Cunha et al., 2016; Fadel et al., 2018; Souza, \& Reinert, 2010) também constataram que a satisfação com o corpo docente é aspecto de destaque na satisfação do aluno.

O curso, como sua grade curricular, teve avaliação satisfatória dos alunos. Esse resultado confirma achados de outros estudos (Cunha et al., 2016; Souza, \& Reinert, 2010), que observaram como elementos proporcionadores de maior satisfação a relação professor-aluno e a grade curricular, além de demonstrarem serem relevantes as formas de organização e transmissão do conteúdo do curso. Outra palavra evidenciada foi o conhecimento, cuja posição de importância demonstra que o estudante espera adquirir o saber necessário para sua formação e não simplesmente concluir uma graduação, ou seja, ela remete ao desejo do discente em sair do curso de fato preparado para exercer sua profissão. Outra palavra importante nas análises é o termo estágio, que se apresenta como responsável por proporcionar ao aluno a oportunidade de praticar o conteúdo aprendido e desenvolver as habilidades necessárias para sua formação. É uma forma de fazer a transição entre a universidade e o trabalho. Para Lima, Paixão, Cândido, Campos e Ceolim (2014), o estágio possibilita o desenvolvimento não só profissional, mas também o pessoal, tornando assim o aluno mais confiante para atuar profissionalmente. A importância de práticas aliadas à teoria no curso foi observada por Santos et al. (2014), em alunos do curso de Medicina, como um fator que favorece a aprendizagem e, consequentemente, a satisfação do aluno com a experiência acadêmica.

Observa-se que as palavras curso e estágio apresentam-se distribuídas no grafo em oposição, mas destacam-se por sua importância na satisfação dos estudantes com o curso. Segundo Lima et al. (2014), a prática do estágio é um momento específico da aprendizagem fundamental à formação do estudante, uma vez que possibilita exercitar na prática os conhecimentos teóricos oferecidos pelo curso, além de proporcionar uma reflexão sobre a ação profissional, fomentando a elaboração de novos conhecimentos. Dessa forma, o estudante que vivencia a experiência do estágio pode transcender os conhecimentos teóricos desenvolvidos em sua formação universitária. Assim, a relação de oposição pode ser justificada pelo fato de o curso ter uma visão mais global e teórica e o estágio ser algo à parte do curso, como uma experiência prática.
Quanto à Insatisfação, o eixo central apresentou a palavra "falta" como a de maior incidência e relacionada a instituição, curso, informações dadas e organização. A insatisfação com professores também é proeminente. Outro tema que se sobressai refere-se aos estágios e à grade curricular. O estudante universitário, no decorrer do curso, se depara com inúmeras dificuldades próprias das dinâmicas de uma IES, que requerem do aluno maior autonomia e capacidade de lidar com esse novo ambiente, novos métodos de estudos e novas relações interpessoais (Oliveira, Wiles, Fiorin, \& Dias, 2014).

Em estudos realizados por Richartz, Ensslin, Valomorbida e Cardoso (2017), em que comparam três IES acerca dos elementos de insatisfação com o curso, indica-se a grade curricular como a mais apontada entre os estudantes. São múltiplos aspectos considerados pelos alunos, como: disciplinas desatualizadas em relação ao mercado de trabalho e índices de reprovação em algumas disciplinas que são pré-requisitos para outras, o que ocasiona atrasos no processo de formação e, consequentemente, desestrutura-se a grade curricular dos períodos posteriores. Para os autores, uma IES se apresenta como objeto de busca por parte do estudante mas, ao ingressar, ele depara-se com uma realidade diferente daquela idealizada ou apresentada pela própria instituição.

Nos estudos de Ramos et al. (2015) com estudantes de um curso de enfermagem em IES pública do Sul, indica-se a dimensão Satisfação com a Instituição como aquela que obteve maiores médias de insatisfação. Sob a perspectiva dos alunos, a insatisfação deve-se à baixa qualidade dos eventos oferecidos pela instituição à sala de aula e à precariedade das áreas comuns, caracterizadas como de baixo conforto. Alunos que buscavam conhecimento por meio de atividades extracurriculares obtiveram um maior índice de insatisfação com a Instituição. Isso permite considerar que quanto maior o interesse sobre temas referentes ao curso escolhido, mais se percebe os déficits encontrados na instituição e no curso. Resultados similares foram encontrados na pesquisa realizada por Carmo et al. (2011) com cerca de 385 estudantes de uma universidade pública do Amazonas. Quando avaliada em sua estrutura e em sua capacidade de corresponder às necessidades básicas de ensino aprendizagem, $62 \%$ dos alunos posicionaram-se como muito insatisfeitos e/ou insatisfeitos.

A percepção dos alunos a respeito da universidade é de fundamental importância para seu melhor 
desenvolvimento e bem-estar. Em relação a satisfação acadêmica, a estrutura física oferecida pode ser um fator de maior ou menor satisfação com o curso (Campos et al., 2014). Em uma universidade pública do Rio Grande do Norte, cerca de 650 alunos participaram de uma pesquisa cuja finalidade foi identificar o nível de satisfação do estudante com a instituição. Dos itens consultados, os de maior predominância foram a carência de segurança no campus, o escasso acervo bibliográfico, os serviços e informações oferecidos pelo site e a pontualidade dos professores. Para Cunha et al. (2016), é fundamental para o aluno o interesse dos professores no rendimento e no crescimento dos discentes. Quanto mais o estudante percebe nos professores atitudes de comprometimento e qualidade acadêmica, mais satisfeito e comprometido com o curso ele se torna.

A vivência universitária requer, dentre tantas demandas, o tempo de estudo e pesquisa. Um dado que interfere na qualidade da satisfação do estudante universitário refere-se à sobrecarga de atividades acadêmicas, profissionais e/ou pessoais (Ramos et al., 2015). Mesmo as atividades extracurriculares são apontadas como fator de insatisfação e são consideradas pelos alunos apenas como cumprimento de carga horária.

É possível observar a relação de complementaridade entre as palavras professor e alunos, o que corrobora outros estudos (Camargos et al., 2006; Cunha et al., 2016; Douglas et al., 2006; Souza, \& Reinert, 2010) em que o professor é apontado como elemento determinante de forma positiva ou negativa na satisfação do estudante com o curso, tanto com relação à experiência docente e ao domínio dos conteúdos ministrados, quanto à transmissão de conhecimentos e à interação com a turma.

\section{Considerações Finais}

Estudos sobre satisfação de estudantes universitários têm propiciado descobertas importantes que facilitam o processo de permanência e crescimento por parte do alunado universitário, ao apontarem para as fragilidades e deficiências nas IES. As demandas que surgem dessa interação propiciam uma melhor compreensão em termos de vivências nas IES, acarretando a busca de soluções e bem-estar e o crescimento dos estudantes.
Com este estudo, percebe-se uma dupla função da percepção do trabalho do professor; se por um lado é valorizado em sua prática pedagógica, relacional e responsável para uma eficiente transição para o mercado de trabalho; por outro, são criticados pela falta de didática.

Ainda no que tange a satisfação com o curso, por meio deste estudo, detectou-se que, para os estudantes, é de grande importância que na grade curricular haja disciplinas cujos conteúdos sejam apresentados de forma a serem posteriormente utilizados em sua prática profissional. A grade curricular bem articulada impacta positivamente também na percepção que os alunos têm da IES. Para os estudantes, isso reflete a importância que a universidade atribui ao curso e ao aluno.

Em sentido oposto, a insatisfação com o curso ocorre quando a IES proporciona atividades extracurriculares de baixa qualidade, estrutura precária no campus, pouca oferta de estágios e falta de informação sobre carreira e necessidades pessoais. Consequentemente, podem gerar insegurança sobretudo quanto a perspectivas para o mercado de trabalho. Assim, a atualização curricular constante, as melhorias estruturais, o aumento da oferta de estágios e a acessibilidade a informações solicitadas pelos estudantes, aumentam o nível de satisfação com o curso.

Com os resultados encontrados nesta pesquisa, abre-se inúmeras possibilidades para uma atuação mais efetiva por parte das IES. Os dados encontrados podem ser facilitadores de ações que suprimam, em parte, as lacunas encontradas e possam proporcionar uma maior e melhor percepção por parte dos alunos a respeito da universidade.

Entre as limitações deste estudo, figuram o fato de não terem sido exploradas as diferenças entre as áreas de inserção dos estudantes, assim como as falas de alunos de diferentes classes sociais, o que foi explorado em outros estudos em que as outras perguntas do questionário foram analisadas. Também pode-se evidenciar situações que favoreçam a satisfação com o curso. Estudos que comparem etapas distintas dos cursos, os motivos da escolha e um número maior de participantes podem trazer novas percepções que colaborem com as universidades e, consequentemente, os que delas fazem parte. 


\section{Referências}

Alcântara, V. de C., Luiz, G. V., Ferreira, A. C., \& Teodoro, S. A. S. (2012). Dimensões e determinantes da satisfação de alunos em uma instituição de ensino superior. REMark - Revista Brasileira de Marketing, 11(3), 193-220. https://doi.org/10.5585/remark.v1 li3.2444

Associação Brasileira de Empresas de Pesquisa. (2018). Associação Brasileira de Empresas de Pesquisa [Website]. http://www.abep.org.

Bordin, D., Saliba, N. A., Fadel, C. B., de Souza, J. A., Garbin, A. J. I., \& Garbin, C. A. S. (2016). Satisfação com a vivência acadêmica entre concluintes de uma universidade pública brasileira [trabalho apresentado]. $6^{\circ}$ Congresso da FOA-UNESP, Araçatuba, SP, Brasil. https://doi.org/10.21270/archi.v5i0.1334

Camargo, B.V., \& Justo, A. M. (2013). IRAMUTEQ: um software gratuito para análise de dados textuais. Temas em Psicologia, 21(2), 513-518.

Camargos, M. A., Camargos, M. C. S., \& Machado, C. J. (2006). Análise das preferências de ensino de alunos de um curso superior de administração de Minas Gerais. Revista de Gestão da USP, 13(2), 1-14.

Campos, D. F., Martins, L. D. S., \& Neto, M. L. (2014). Qualidade dos serviços na educação superior: uma visão comparativa entre dois cursos de graduação [trabalho apresentado]. Simpósio de Administração da Produção, Logística e Operações Internacionais, São Paulo, Brasil.

Carmo, Y. A. do., Silva, V. E., Miranda, V. da S., Rabelo, L. M. B., \& Guimarães, M. da. G. V. (2011). Análise de satisfação dos alunos da faculdade de estudos sociais da Universidade Federal do Amazonas [trabalho apresentado]. Congresso Virtual Brasileiro de Administração Online, Brasil.

Cunha, P. R., Gomes, G., \& Beck, F. (2016). Satisfação dos Estudantes do Curso de Ciências Contábeis: Estudo em Universidades Públicas de Santa Catarina. Revista Contabilidade Vista e Revista, 27(1), 42-62.

Douglas, J., Douglas, A., \& Barnes, B. (2006). Measuring student satisfaction at a UK university. Quality Assurance in Education, 14(3), 251-267. https://doi.org/10.1108/09684880610678568

Fadel, C. B., Souza, J. A., Bordin, D., Garbin, C. A. S., Garbin, A. J. I., \& Saliba, N. A. (2018). Satisfação com a vivência acadêmica entre concluintes de uma universidade pública brasileira. Revista Gaúcha de Odontologia, 66(1), 50-59. https://doi.org/10.1590/1981-863720180001000073261

Fernandes, B. A. O. F., Silva, J. M. C. A., Ravena, M., Martins, C. M., \& Gomes, S. C. (2017). Elementos constitutivos da satisfação dos acadêmicos de graduação dos cursos de administração e ciências contábeis da Universidade Federal do Paraná [trabalho apresentado]. XX SEMEAD - Seminários em Administração, São Paulo, SP, Brasil.

Gomes, G., Dagostini, L., \& Cunha, P. R. (2013). Satisfação dos estudantes do curso de Ciências Contábeis: estudo em uma faculdade do Paraná. Revista da Faculdade de Administração e Economia, 4(2), 102-123. http://dx.doi.org/ 10.15603/2176-9583/refae.v4n2p102-123

Hirsch, C. D. (2015). Satisfação acadêmica dos estudantes de enfermagem com o curso de graduação [Tese de Doutorado em Enfermangem, Universidade Federal do Rio Grande do Sul].

Hirsch, C. D., Barlem, E. L. D., Barlem, J. G. T., Silveira, R. S., \& Mendes, D. P. (2015). Fatores preditores e associados à satisfação dos estudantes de enfermagem. Acta Paulista de Enfermagem, 28(6), 566-572. https://doi.org/ $10.1590 / 1982-0194201500093$

Hirsch, C. D, Barlem E. L. D, Tomaschewski-Barlem, J. G., Dalmolin, G. L., Pereira, L. A., \& Ferreira, A. G. (2016). Cross-cultural adaptation and validation of the Nursing Student Satisfaction Scale for use with Brazilian nursing students. Revista Latino-Americana de Enfermagem, 24. https://doi.org/10.1590/1518-8345.1053.2776

Javed, N., \& Bzmi, N. (2018). Effects of teaching style on student's satisfaction through the mediating role of teacher's behavior and qualification. Sumerianz Journal of Education, Linguistics and Literature, 1(4), 96-105.

Justo, A. M., \& Camargo, B. V. (2014). Estudos qualitativos e o uso de softwares para análises lexicais. In C. Novikoff, S. R. M. Santos \& O. B. Mithidieri (Orgs.). Caderno de Artigos: X SIAT \& II Serpro (pp. 37-54). Universidade do Grande Rio "Professor José de Souza Herdy". https://lageres.wordpress.com/

Lima, T. C. D., Paixão, F. R. C., Cândido, E. C., Campos, C. J. G., \& Ceolim, M. F. (2014). Estágio curricular supervisionado: análise da experiência discente. Revista Brasileira de Enfermagem, 67(1), 133-140. https://doi.org/ 10.5935/0034-7167.20140018

Martins, F. (1998). A satisfação acadêmica: Construção de uma escala (pp. 188-193) [trabalho apresentado]. IV Congresso Galaico-Português de Psicopedagogia, Braga, Portugal. 
Nascimento, L. C. N., Souza, T. V., Oliveira, I. C. S., Moraes, J. R. M. M., Aguiar, R. C. B., \& Silva, L. F. (2018). Saturação teórica em pesquisa qualitativa: relato de experiência na entrevista com escolares. Revista Brasileira de Enfermagem, 71(1), 243-248. https://doi.org/10.1590/0034-7167-2016-0616

Oliveira, C. T., Wiles, J. M., Fiorin, P. C., \& Dias, A. C. (2014). Percepções de Estudantes universitários sobre a relação professor-aluno. Revista Quadrimestral da Associação Brasileira de Psicologia Escolar e Educacional, 18(2), 239-246. https:// doi.org/10.1590/2175-3539/2014/0182739

Pinto, N. G. M., Quadros, M. R. C., Cruz, F. V., \& Conrad, C. C. (2016). Satisfação acadêmica no Ensino Superior brasileiro: uma análise das evidências empíricas. Revista Brasileira de Ensino Superior, 3(2), 3-17. https://doi.org/ 10.18256/2447-3944.2017.v3i2.1600

Ramos, A. M., Barlem, J. G. T., Lunardi, V. L., Barlem, E. L. D., Silveira, R. S., \& Bordignon, S. S. (2015). Satisfação com a experiência acadêmica entre estudantes de graduação em enfermagem. Texto \& Contexto - Enfermagem, 24(1), 187-195. https:// doi.org/10.1590/0104-07072015002870013

Richartz, M. S., Ensslin, S. R., Valomorbida, S. M. L., \& Cardoso, T. L. (2017). Satisfação de Discentes no Curso de Ciências Contábeis em Universidades Públicas. Sociedade, Contabilidade e Gestão, 12(2), 46-62. https://doi.org/ 10.21446/scg_ufrj.v12i2.13405

Santos, M. R., Goulart, A. L., Miyoshi, M. H., \& Santos, A. M. N. (2014). A importância de um Questionário de Avaliação de Unidade Curricular. Revista Brasileira de Educação Médica, 38(2),190-197.

Schleich, A. L. R., Polydoro, S. A. J., \& Santos, A. A. A. (2006). Escala de satisfação com a experiência acadêmica de estudantes de ensino superior. Avaliação Psicológica, 5(1), 11-20.

Silva, R. C. (2015). Educação: a outra qualidade. UNIMEP.

Soares, A. P., Vasconcelos, R. M., \& Almeida, L. S. (2002). Adaptação e satisfação na Universidade: Apresentação e validação do Questionário de Satisfação Académica (QSA). In A. S. Pouzada, L. S. Almeida \& R. M. Vasconcelos (Eds.), Contextos e dinâmicas da vida académica (pp. 153-165). Guimarães; Universidade do Minho.

Souza, S. A., \& Reinert, J. N. (2010). Avaliação de um curso de ensino superior através da satisfação/insatisfação discente. Avaliação, 15(1), 159-176. https://doi.org/10.1590/S1414-40772010000100009

\section{Adriana Benevides Soares}

Psicóloga, doutora pela Universidade de Paris XI, pós-doutora pela UFSCar e pela USF. Professora da Universidade Salgado de Oliveira (UNIVERSO), São Gonçalo - RJ. Brasil. Professora da Universidade do Estado do Rio de Janeiro (UERJ), Rio de Janeiro - RJ. Brasil.

E-mail: adribenevides@gmail.com

(1) https://orcid.org/0000-0001-8057-6824

\section{Isabela da Silva Rodrigues}

Psicóloga, mestre em Psicologia Social pela Universidade Salgado de Oliveira (UNIVERSO), São Gonçalo - RJ. Brasil. E-mail: rodrigues.isabelasilva@gmail.com

(1) https: / / orcid.org/ 0000-0002-3263-5575

\section{Giselle Glória Balbino dos Santos}

Pedagoga, mestre em Psicologia Social pela Universidade Salgado de Oliveira (UNIVERSO), São Gonçalo - RJ. Brasil. E-mail: gbsantos25@gmail.com

(1) https://orcid.org/0000-0001-6738-3059

\section{Claudio de Almeida Lima}

Psicólogo, mestre em Psicologia Social pela Universidade Salgado de Oliveira (UNIVERSO), São Gonçalo - RJ. Brasil.

E-mail: limaclaudio74@hotmail.com

(1) https://orcid.org/0000-0002-5404-7029 
Psicologia: Ciência e Profissão 2021 v. 41, e220715, 1-12.

Endereço para envio de correspondência:

Universidade Salgado de Oliveira. Rua Mal. Deodoro, 217, bloco A, Centro. CEP: 24030-060. Niterói - RJ. Brasil.

Recebido 01/03/2019

Aceito 05/11/2020

Received 03/01/2019

Approved 11/05/2020

Recibido 01/03/2019

Aceptado 05/11/2020

Como citar: Soares, A. B., Rodrigues, I. S., Santos, G. G. B., Lima, C. A. (2021). A Satisfação de Estudantes Universitários com o Curso de Ensino Superior. Psicologia: Ciência e Profissão, 41, 1-12. https://doi.org/ $10.1590 / 1982-3703003220715$

How to cite: Soares, A. B., Rodrigues, I. S., Santos, G. G. B., Lima, C. A. (2021). The Satisfaction of University Students with Higher Education Courses. Psicologia: Ciência e Profissão, 41, 1-12. https://doi.org/10.1590/ 1982-3703003220715

Cómo citar: Soares, A. B., Rodrigues, I. S., Santos, G. G. B., Lima, C. A. (2021). La Satisfacción de los Universitarios con el Curso de Educación Superior. Psicologia: Ciência e Profissão, 41, 1-12. https://doi.org/10.1590/ 1982-3703003220715 